\title{
Physical Model and Mesoscale Simulation of Mortar and Concrete Deformations under Freeze-Thaw Cycles
}

\author{
F. Gong, E. Sicat, Y. Wang, and T. Ueda \\ Department of Civil Engineering, Hokkaido University \\ D. Zhang \\ Department of Civil Engineering, Zhejiang University
}

\begin{abstract}
The degradation of concrete material under multiple freeze-thaw cycles is an important issue for structures in cold and wet regions. This paper proposed a physical and mechanical model to explain the deformation behavior observed in previous experiments, from internal pressure calculation to mesoscale simulation, and for both closed and open freeze-thaw tests. Three kinds of internal pressures are considered in this study: hydraulic pressure due to ice volume expansion, crystallization pressure, and cryosuction pressure due to liquid-ice interface. The hydraulic pressure model combines Power's model with poromechanical theories, which can well explain the reverse phenomenon (from expansion to contraction) observed in the closed test. The total internal pressure will be applied in a discrete numerical method (Rigid Body Spring Model) to simulate the deformation during each cycle, as well as the unrecoverable cracking (residual strain) at the end of each cycle. The constitutive laws are also modified considering the features of those internal pressures. Finally, the deformation behaviors of mortar, mortar-aggregate interface (closed test, 30 cycles), and the concrete (open test, 300 cycles) are simulated and compared with experiment measurements, which are found in a satisfactory agreement.
\end{abstract}

\section{INTRODUCTION}

Frost damage is an important issue for concrete structures, and has been studied for several decades. Kaufmann (2002) developed a qualitative sequential damage model, separating a freeze-thaw cycle into five phases and discussed it in detail. Fagerlund (2002) discussed the different effects between open and closed freeze-thaw tests and the effect of saturation degree. For the open test, the deformation measure by Hasan, Okuyama, Sato, and Ueda (2004) up to 300 cycles showed a continually increasing behavior. However, recent closed tests by the authors up to 30 cycles showed a different phenomenon: there was expansion during the first few cycles, but was converted to contraction as the number of cycles increased (Sicat, Gong, Zhang, \& Ueda, 2013). Although the damage of material would affect the measured deformation, this change of tendency can only be explained by the change of forces: from positive forces dominant to negative forces dominant. Thus, a more comprehensive stress model is needed to explain this complex strain behavior.

The stress that causes frost damage is believed to be due to the hydraulic pressure at the beginning. Powers (1949) developed the hydraulic pressure model based on Darcy's law, which is a time-dependent approach. Recent studies discussed that other than hydraulic pressure, the crystallization pressure is another mechanism (Scherer \& Valenza, 2005), because damage was still observed in partially saturated cases in which hydraulic pressure could be avoided. In addition, due to the thermodynamic equilibrium between three phases of moisture, there is always cryosuction pressure in the unfrozen water. Another static model by Coussy and Monteiro's (2008) was developed based on the poromechanics, but did not consider the hydraulic flow and pressure release (like Powers' model). By combining Powers' model with poromechanics, both deformation compatibility and pressure release can be taken into consideration, as well as the shrinkage by cryosuction pressure.

After achieving a more flexible and comprehensive pressure model, the estimated internal forces will be applied on Rigid Body Spring Model (RBSM), a discrete numerical model to simulate the deformation and damage (Kawai, 1977). Mesoscopic analysis is relatively more precise to simulate each component (mortar, aggregate, and their interface) in the concrete. In addition, this discrete model has the advantage of showing the cracking and failure mode. Previous researchers have used RBSM to simulate the concrete materials under static load (Nagai, Sato, \& Ueda, 2004), fatigue load (Matsumoto, Sato, Ueda, \& Wang, 2008), and also the mechanical properties of frost-damaged concrete (Ueda, Hasan, Nagai, Sato, \& Wang, 2009). However, there is still no simulation on 
the degradation process itself, with quantitative input of internal forces.

Therefore, this article will present the modified internal pressure model, which can explain the previous observations in both closed and open tests, and then apply the estimated internal forces on the RBSM program, and finally, the simulated results will be compared with the macro experiment phenomenon.

\section{INTERNAL PRESSURES}

\subsection{Hydraulic pressure}

There are two main hydraulic theories for the freezing process in porous cement-based materials. One is proposed by Powers (1949), which aims to determine the suitable spacing factor of the air bubbles to avoid frost damage in the concrete. In his model, it was assumed that liquid water can be expelled into the entrained air voids once ice forms in the surrounding material. According to Darcy's law, a pressure gradient is a must to drive such kind of water flow; thus hydraulic pressure generates. And a simple expression is:

$$
p_{h}=q \cdot \frac{\eta}{k} \cdot d
$$

where $p_{\mathrm{h}}$ is the local hydraulic pressure in pores, $k$ is the permeability of porous body $\left(\mathrm{m}^{2}\right), \eta$ is the viscosity of liquid water $(\mathrm{Pa} \cdot \mathrm{s})$, and $d$ is the equivalent distance from the empty voids. $q$ is the water flow $(\mathrm{m} / \mathrm{s})$, which depends on the volume changing rate during ice formation.

Other than Powers' model, Coussy and Monteiro (2008) neglected the water flow and proposed a poromechanical model for saturated porous materials in which the increased volume can be balanced by the compression of water and ice, giving liquid pressure as:

$$
p_{h} \approx 0.09 \cdot \frac{\psi_{C}}{\psi_{C} / K_{C}+\left(1-\psi_{C}\right) / K_{L}}
$$

Where $\psi_{c}$ is the normalized ice content; therefore $\psi_{L}=1-\psi_{C}$ is the liquid water content. $K_{C}$ and $K_{L}$ are the bulk moduli of the ice and liquid, respectively. This model also describes an ideal condition, which is based on the assumption that the hydraulic pressure resulting from the volume change cannot escape (sealed condition or the air voids are very far apart). However, in reality, both water flow and selfcompression will exist depending on the distribution of empty pores (like entrained air) and permeability of the materials. Thus, a comprehensive expression would be:

$$
0.09 \phi \psi_{C}-Q=\varepsilon_{p}-\phi \psi_{C} \varepsilon_{C}-\phi \psi_{L} \varepsilon_{L}
$$

where $Q$ represents the water flow by Powers' model, which only accounts for part of the increased volume; $\phi$ is porosity and $\varepsilon$ is the volume strain with subscripts $p, C$, and $L$ for porous body, ice (crystal) and water (liquid), respectively. And the other part is balanced by the deformation of materials and water (liquid and solid). The time differential form of Equation (3) becomes:

$$
0.09 \phi \dot{\psi}_{C}-\frac{A}{V} \cdot q=\dot{\varepsilon}_{p}-\phi \psi_{C} \dot{\varepsilon}_{C}-\phi \psi_{L} \dot{\varepsilon}_{L}
$$

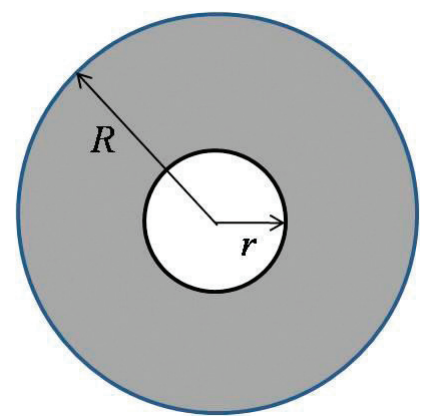

Figure 1. Equivalent empty space and influential volume.

where $A=4 \pi r_{E}^{2}, V=\frac{4}{3} \pi\left(R_{E}^{3}-r_{E}^{3}\right) \cdot r_{E}$ and $R_{E}$ are the equivalent radii of the entrained air (or the empty pores) and the influential volume. $r_{\mathrm{E}}$ can be chosen as the weighted mean value of empty volume, according to the pore size distribution $v(r)$ :

$$
r_{E}=\int_{\infty}^{r_{0}} r \cdot v(r) d r / \int_{\infty}^{r_{0}} v(r) d r
$$

Where $r_{0}$ is the critical radius between empty and filled pores. $r_{\mathrm{E}}$ and $R_{\mathrm{E}}$ should also satisfy:

$$
r_{E}^{3} / R_{E}^{3}=(1-S r) \cdot \phi
$$

where $S r$ is the saturation occupied by ice and liquid, equals to $\psi_{L}+\psi_{C}$. The $q$ in Equation (4) can be approximately written as:

$$
q=\frac{k}{\eta} \cdot \frac{\Delta p_{h}}{\Delta x}\left(\Delta p_{h}=p_{h}, \Delta x=\left(R_{E}-r_{E}\right) / 2\right)
$$

Actually, the pressure gradient is not exactly as Equation (7), but since here we discuss this problem on a larger scale, only the equivalent or average value is needed. At the same time, due to this pressure $p_{\mathrm{h}}$, the material will expand while the liquid and ice will be compacted, that is:

$$
\left\{\begin{array}{l}
\varepsilon_{p}=b p_{h} / K_{p} \\
\varepsilon_{C}=-p_{h} / K_{C} \\
\varepsilon_{L}=-p_{h} / K_{L}
\end{array}\right.
$$

where $b=2 \phi /(1+\phi)$ is the Biot coefficient (Coussy, 2004) and $K_{p}$ is the bulk modulus of porous body. 
Here linear behavior was assumed for all these three components for convenience. Then take Equations (7) and (8) into Equation (4), it becomes:

$0.09 \phi \dot{\psi}_{C}-\frac{A}{V} \cdot \frac{k}{\eta} \cdot \frac{2}{R_{E}-r_{E}} p_{h}=\left(\frac{b}{K_{p}}+\frac{\phi \psi_{C}}{K_{C}}+\frac{\phi \psi_{L}}{K_{L}}\right) \cdot \dot{p}_{h}$

Then by solving Equation (9), hydraulic pore pressure $\left(p_{\mathrm{h}}\right)$ can be obtained during the whole freeze-thaw cycle.

The ice forming rate $\dot{\psi}_{C}=\left(d \psi_{C} / d T\right) \cdot(d T / d t)$ can be determined by the cooling rate and the ice content at each temperature, for example, Sun \& Scherer's DSC data (2010a). The viscosity $\eta$ in Equation (9) is also depending on the temperature, which gives (Coussy, 2005)

$$
\eta=\eta_{0} \cdot 1.6 \times 10^{-2} \times \exp \left(\frac{509.53}{123.15+T}\right)
$$

where $\eta_{0}=0.0018 \mathrm{~Pa} \cdot s$ is the viscosity of water at the temperature of $0^{\circ} \mathrm{C}$. In addition, once the pore network is partially blocked by the formed ice, the permeability $k$ will also decrease. According to Coussy's paper (2005), the modified form of van Genuchten equations for concrete materials are:

$$
\begin{gathered}
k_{r}=\frac{k}{k_{0}}=S_{L}^{0.5}\left[1-\left(1-S_{L}^{\frac{1}{m}}\right)^{m}\right]^{2} \\
S_{L}=\left[1+\left(\frac{R_{\star}}{R}\right)^{1 /(1-m)}\right]^{-m}
\end{gathered}
$$

where $k_{0}$ is the saturated permeability (without ice), $k_{r}$ is the relative value showing the reduction due to ice formation, $S_{L}=\psi_{L}$ is the liquid saturation degree, and $R$ is the critical pore size for freezing under different temperatures. $R_{*}=4.26 \mathrm{~nm}$ for cement-based materials, a parameter related to the percolation (Coussy, 2005). Then, the parameter $m$ can be determined according to Sun's DSC data (2010b), and it is approximately 0.5 . Therefore, the relative permeability by liquid saturation should be:

$$
k_{r}=\frac{k}{k_{0}}=S_{L}^{0.5}\left[1-\left(1-S_{L}^{2}\right)^{0.5}\right]^{2}
$$

\subsection{Cryosuction and crystallization pressure}

Due to the surface tension, there is a pressure difference between liquid and crystal on the crystal/ liquid interface, and also a difference between liquid and gas on the liquid/vapor interface. If assuming the pressure of the gas is the same as the ambient pressure (0), then the cryosuction pressure is always negative and depends on the temperature (Sun \& Scherer, 2010a):

$$
p_{I}=\psi_{L} \cdot \Delta S_{f v}\left(T-T_{0}\right)
$$

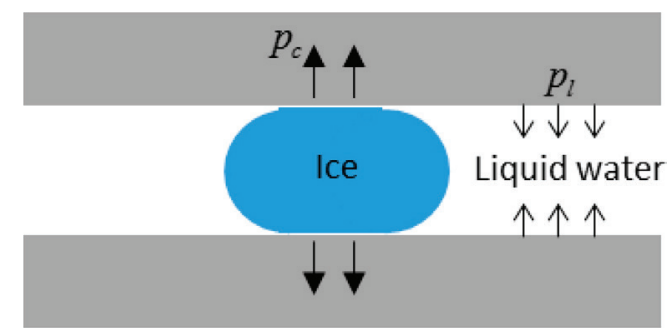

Figure 2. Crystallization pressure and cryosuction pressure in a cylindrical pore.

where $T_{0}=0^{\circ} \mathrm{C}, \Delta S_{f v} \approx 1.2 \mathrm{~J} / \mathrm{cm}^{3} \cdot \mathrm{K}$ is the molar entropy of fusion. The crystallization pressure acting on the pore wall is always accompanied by the cryosuction pressure (Figure 2):

$$
p_{c}=-\psi_{C} \cdot(1-\lambda) \Delta S_{f v}\left(T-T_{0}\right)
$$

where $\lambda$ is the pore shape factor ( 0 for sphere pore and 0.5 for cylindrical pore) (Sun \& Scherer, 2010a). And here it is regressed based Sun's data as:

$$
\lambda=-0.0095 T+0.125
$$

Therefore, the crystallization pressure can be calculated as:

$$
p_{c}=-\psi_{C} \cdot(0.875+0.0095 T) \Delta S_{f v}\left(T-T_{0}\right)
$$

\subsection{Experimental verification}

The mortar specimens in the closed test (Sicat et al., 2013) used ordinary Portland cement with density of $3.14 \mathrm{~g} / \mathrm{cm}^{3}$, fine aggregate which is $1.2 \mathrm{~mm}$ or less in size with density of $2.67 \mathrm{~g} / \mathrm{cm}^{3}$ at $1467.6 \mathrm{~kg} / \mathrm{m}^{3}$ of concrete without air entraining agent to promote damage. Mix proportion for specimens is 1:2:6 (water:cement:fine aggregate). After curing, specimens were cut into size of $40 \mathrm{~mm} \times 40 \mathrm{~mm} \times 2 \mathrm{~mm}$ (see Figure 3(a)). Specimens were submerged under water until mass was constant to attain full saturation. Finally, the specimens were sealed with vinyl tape to prevent water uptake or loss. The preparation of the specimens is shown in Figure $3(a)$ and (b). The size distribution of entrapped air can be obtained from image anlysis (Figure 3(c)). Figure3(d) shows the temperature history of each cycle.

Adopting the parameters listed in Table 1 to the proposed model, the three kinds of pore pressure can be calculated (Figure 4). Crystallization and cryosuction pressures always coexist and the sum of the two is less than 0. Hydraulic pressure is closely related to the permeability $\left(k_{0}\right)$; for the first freezethaw cycle, the permeability of undamaged material can be assumed. Thus, for closed test without water 
uptake, the hydraulic pressure will reduce gradually. The total pressure in Figure 4 is still positive, which means expansion occurs at the beginning. However, as the damage is cumulated, the permeability will increase; this was also proved experimentally by Yang, Weiss, and Olek (2006). During this process, the crystallization pressure and cryoscution pressure is considered unchanged. Still using Equation (9), but with different magnitudes of permeability, the hydraulic pore pressure is shown in Figure 5.

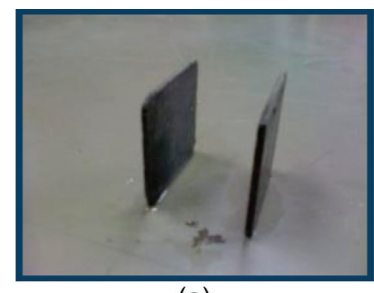

(a)

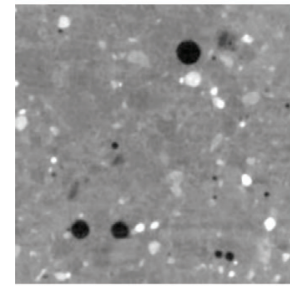

(c)

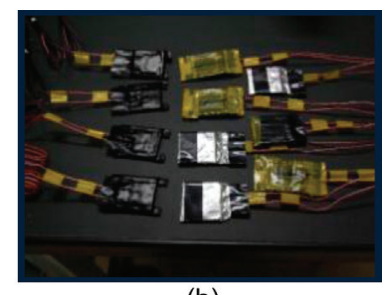

(b)

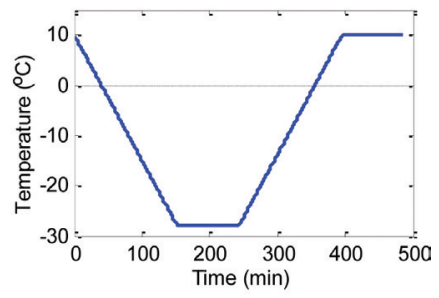

(d)
Figure 3. Outline of the experiment (a) $40 \mathrm{~mm} \times 40 \mathrm{~mm} \times 2 \mathrm{~mm}$ specimens; (b) sealed specimens; (c) X-ray CT scanning $(6.2 \mathrm{~mm} \times 6.2 \mathrm{~mm}) ;(\mathrm{d})$ temperature history of one cycle.

Table 1. Experimental and empirical parameters for mortar in closed test.

\begin{tabular}{lc}
\hline \multicolumn{1}{c}{ Type (w/c) } & Mortar (0.5) \\
\hline Mix proportion by weight (w:c:a) & $1: 2: 6$ \\
Water saturated (vacuum saturated) & $0.228(0.238) \mathrm{g} / \mathrm{cc}$ \\
Real water saturation & $95.8 \%$ \\
Critical radius $r_{0}$ (by X-ray CT scanning) & $1.7 \times 10^{-4} \mathrm{~m}$ \\
Lowest temperature & $-28^{\circ} \mathrm{C}$ \\
Elastic Modulus $E$ (measured) & $34 \mathrm{Gpa}$ \\
Saturated permeability (undamaged) $k_{0}$ & $10^{-21} \mathrm{~m}^{2}$ \\
Poisson's ratio of mortar $v$ & 0.2 \\
Bulk modulus of porous body $K_{\mathrm{p}}$ & $18.9 \mathrm{GPa}$ \\
Bulk modulus of ice crystal $K_{\mathrm{C}}$ & $8.8 \mathrm{GPa}$ \\
Bulk modulus of liquid water $K_{\mathrm{L}}$ & $2.2 \mathrm{GPa}$ \\
Critical radius $r_{0}$ (Gong, Zhang, Sicat, & $2 \times 10^{-4} \mathrm{~m}$ \\
$\&$ Ueda, 2013b) & $1.7 \times 10^{-4} \mathrm{~m}$ \\
(by X-ray CT scanning) & $3 \times 10^{-4} \mathrm{~m}$ \\
Equivalent $r_{\mathrm{E}}$ [by Equation $\left.(5)\right]$ & $0.0014 \mathrm{~m}$ \\
Equivalent $R_{\mathrm{E}}$ [by Equation $(6)$ ] &
\end{tabular}

Since the internal pressure is rather difficult to measure directly, especially for cement-based materials, the comparison is between the calculated deformation based on a poroelastic assumption and the measured deformation. Therefore, the strain in one dimension caused by total internal pressure is:

$$
\varepsilon_{x}=\left(\frac{b}{3 K_{p}}\right)\left(p_{h}+p_{l}+p_{c}\right)
$$

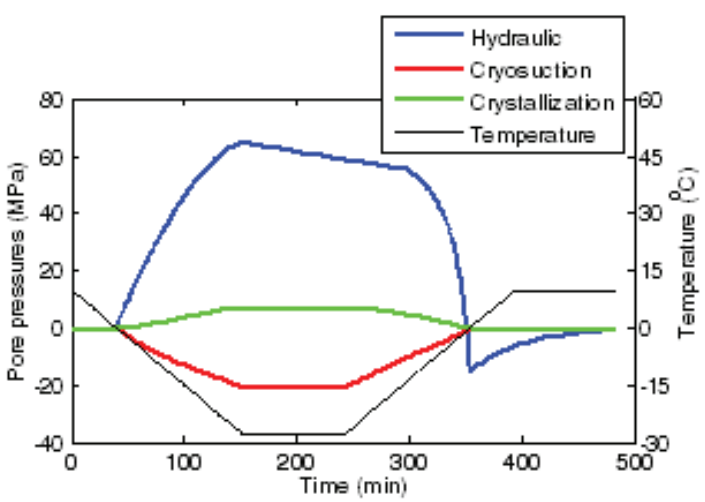

Figure 4. Calculated hydraulic, crystallization, and cryoscution pressures.

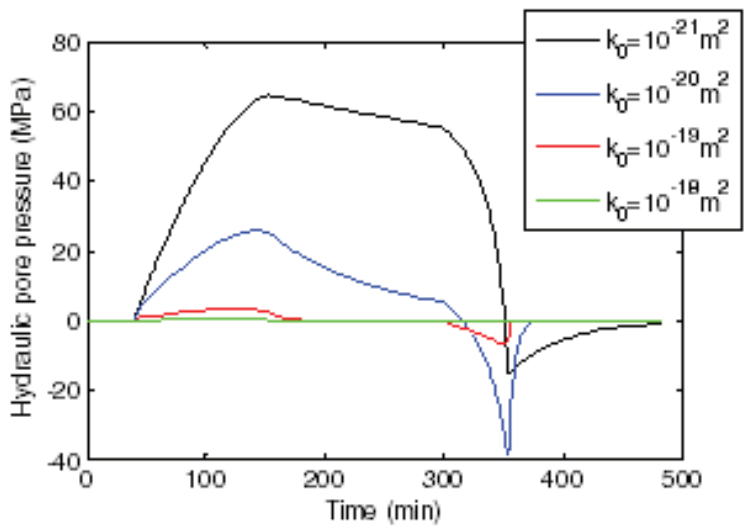

Figure 5. Calculated hydraulic pore pressure in one cycle with different permeability (due to damage).

After choosing the proper value of permeability, the deformation by total pressure can be calculated (Figure 6). In the experiment, the plastic deformation exists because the peak strain already exceeds the limited strain of the tensile strength. The effect of tension-softening behavior will be discussed in the next section, but here, our purpose is just to verify the reliability of pressure model. Therefore, the tensile strain of the first cycle is adjusted within the calculated range [Figure 6(b)]. Other typical cycles are chosen to compare the effect of hydraulic pressure release [Figure 6(b, c)]. Although the curves are not perfectly matched, the results are still convincing, considering the complexity of the measured data.

For the open test, the permeability change is no longer the main influence, but the saturation degree is. It is because the initial saturation degree of open test is 


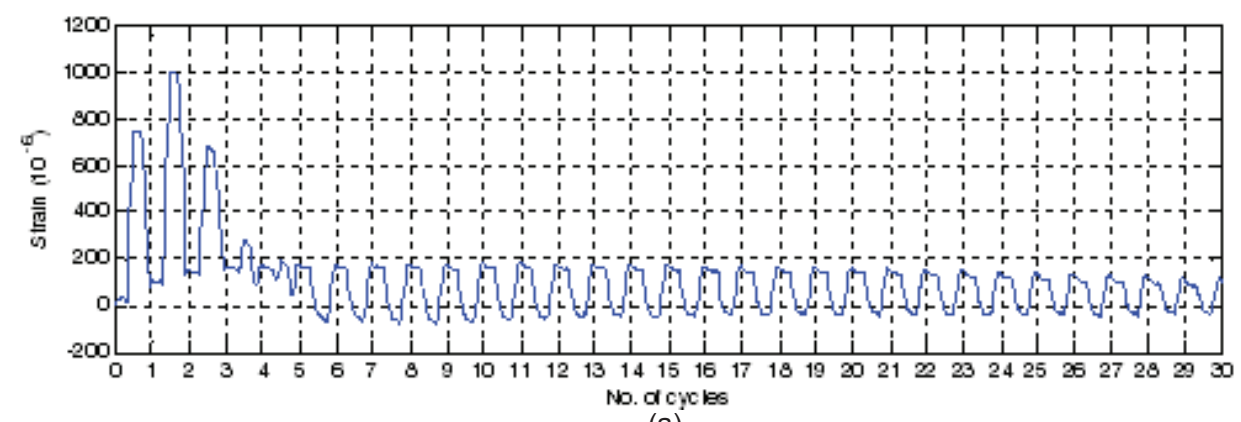

(a)

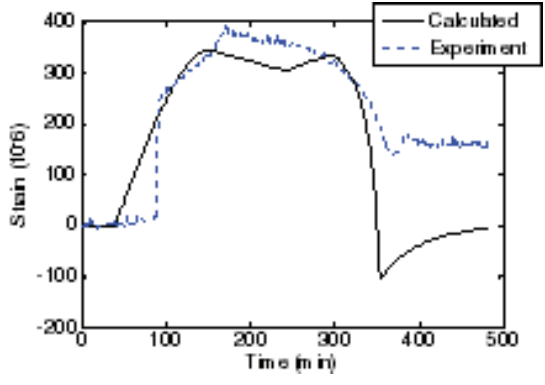

(b)

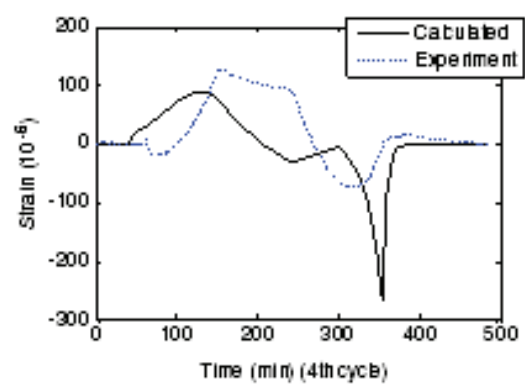

(c)

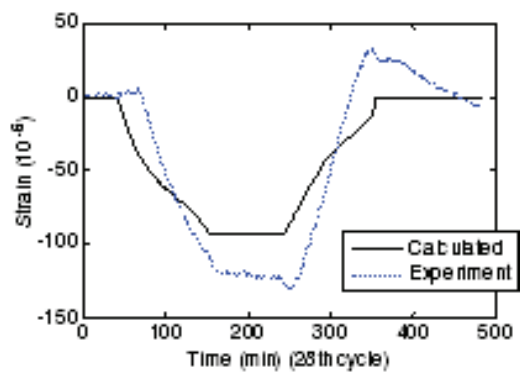

(d)

Figure 6. Calculated and experimental strain (a) Experiment of 30 cycles (Sicat et al., 2013) (b) first cycle, c (c) fourth cycle, $k_{0}=10^{-20} \mathrm{~m}^{2}$ (d) 28 th cycle, $k_{0}=10^{-18} \mathrm{~m}^{2}$.

not very high that the hydraulic pressure is not big enough to cause quick damage. As the number of cycles increase, the saturation degree will gradually increase, resulting in higher hydraulic pressure and larger deformation (Fagerlund, 2002). And this will be simulated in the next section.

\section{MESOSCALE MECHANICAL MODEL}

The Rigid Body Spring Model (RBSM) is a discrete numerical analysis method, which was first developed by Kawai (1977). Unlike the continuum methods, such as Finite Element Method or Finite Difference Method, RBSM is a more proper way to simulate splitting and cracking in cement-based materials like mortar and concrete. Also, compared to other discrete methods like Distinct Element Method, RBSM is more suitable for small deformation and tiny cracks that often occur in concrete structures.

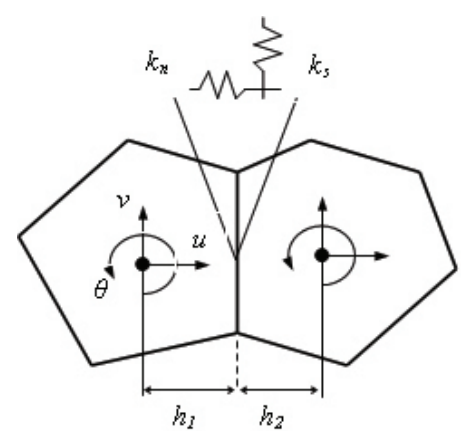

Figure 7. Elements, degree of freedom, and springs.
The analytical model is divided into polyhedron elements, and the mesh is arranged randomly using a Voronoi diagram. Each Voronoi cell represents a mortar or aggregate element in the model. For two adjacent elements, there are two springs connecting them: normal spring and shear spring, which are placed at the boundary of the elements (see Figure 7). Each element has two translational and one rotational degree of freedom at the center of gravity. The constitutive relation for the normal spring under the cyclic internal stress has been discussed in the authors' previous paper (Gong, Sicat, Ueda, \& Zhang, 2013a) as:

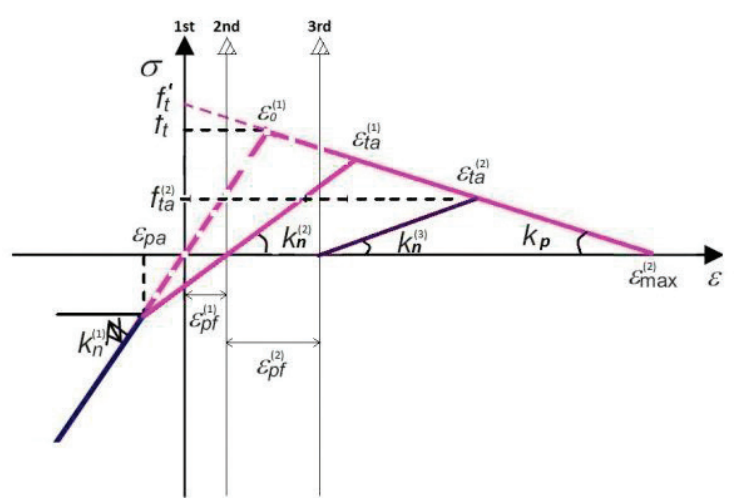

Figure 8. Stress-strain relationship under multiple FTCs.

Figure 8 shows a tension-softening relation, which is more close to the real cases compared to the linear elastic assumption. And compared to the external loading, the internal stress during freeze-thaw itself 
is also dependent on the material's deformation. For example, after exceeding the tensile strength $\left(f_{t}\right)$, the stress equilibrium can still be achieved at $\varepsilon_{\text {ta }}^{(1)}$; this is because as the porous body continuously expand, the hydraulic pressure will decrease linearly as in Figure 9. If the stiffness of a porous body is infinitely large (or we just assume it does not deform), the internal stress should reach the upper bound $\left(\sigma_{0}\right)$. For a fully saturated porous body:

$$
\sigma_{0}=b \cdot p_{h 0}=b \cdot 0.09 \phi \psi_{C} /\left(\frac{\phi \psi_{C}}{K_{C}}+\frac{\phi \psi_{L}}{K_{L}}\right)
$$

Then, if there is a volume strain of the porous body $\varepsilon_{p}\left(=3 \varepsilon_{x}\right)$, the internal stress $\left(\sigma_{w}\right)$ will decrease as:

$$
0.09 \phi \psi_{C}=\left(\frac{\phi \psi_{C}}{K_{C}}+\frac{\phi \psi_{L}}{K_{L}}\right) \cdot \frac{\sigma_{w}}{b}+3 \varepsilon_{x}
$$

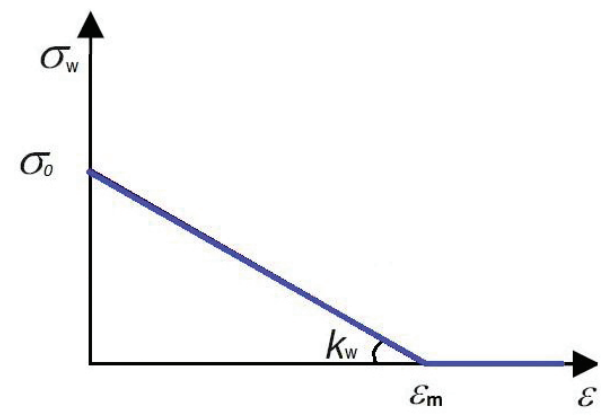

Figure 9. Internal stress regarding the deformation of porous body.

Then, the slope $k_{\mathrm{w}}$ in Figure 9 can be determined as:

$$
k_{w}=\frac{3 b}{\phi\left[\psi_{C} / K_{C}+\psi_{L} / K_{L}\right]}
$$

Then, combining Figures 8 and 9, the equilibrium stress-strain for each normal spring can be determined. Till now, the constitutive relations are not easy to apply to the numerical program because there are two objects (porous body and ice-liquid system) that need to be considered at the same time. If we regard the ice-liquid system as another normal spring, Figures 8 and 9 can be shown as a spring system [see Figure10(a)], and the initial stress $\sigma_{0}$ is applied on the spring of ice and liquid, and finally, two springs will reach an equilibrium. Figure 10(b) shows another case, that is, $\sigma_{0}$ is applied outside and on the two springs, and it can be easily proved that the final stress and strain conditions of the porous body is the same between (a) and (b), but for the ice-liquid system, (a) and (b) are different. Since we are only interested in the degradation of porous body, Figure 10 (a) can be replaced by Figure 10(b), and the stressstrain relation of ice-liquid should also be changed to Figure 11. Then, if using Figure 10(b), the programing is much clearer, we just need to add another stiffness matrix:

$$
\left[K_{p}+K_{w}\right]\{u\}=\{F\}
$$

Where $\left[K_{p}\right]$ and $\left[K_{w}\right]$ are the stiffness matrix of the porous body and ice-liquid system, respectively. And the load boundary $\{F\}$ can be calculated using $\sigma_{0}$ :

$$
\{F\}=[B]^{T}\left\{\sigma_{0}\right\}
$$

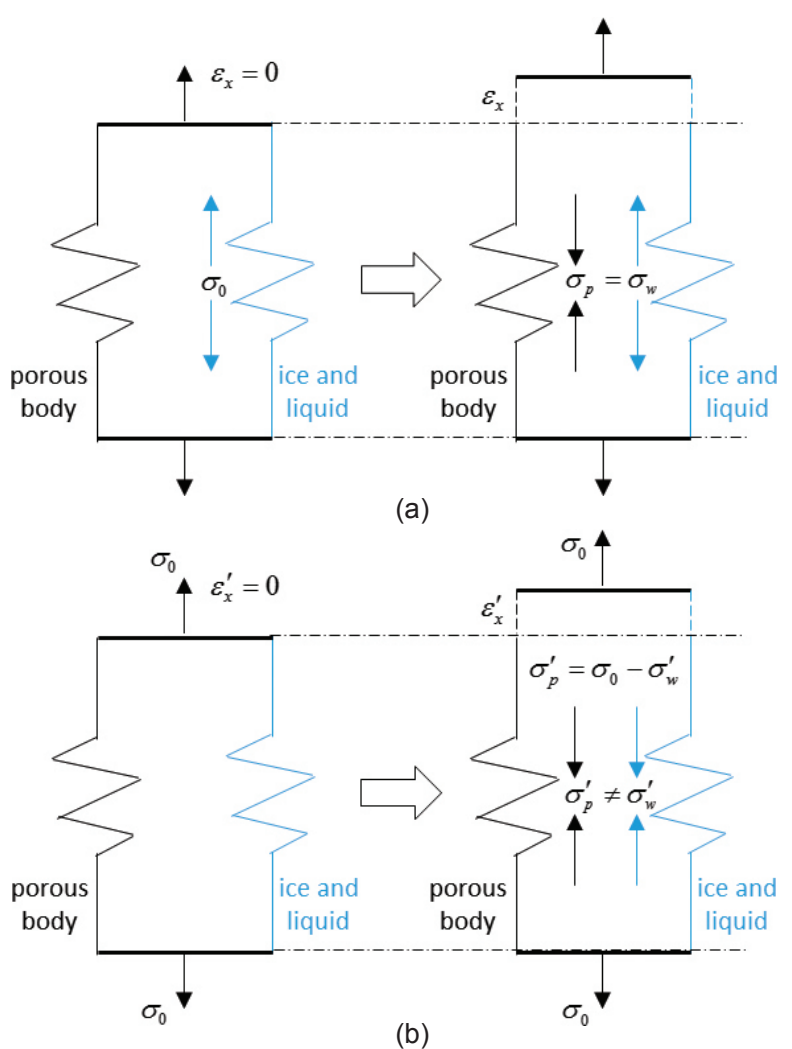

(b)

Figure 10. Parallel spring system (a) input as internal stress (b) input as external stress.

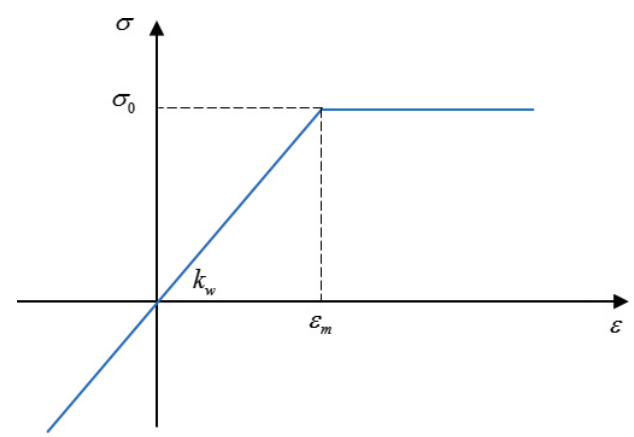

Figure 11. Internal stress regarding the deformation of porous body [for spring in Figure10 (b)].

For the normal springs of porous body, a normal distribution was also assumed for the tensile 
strength to increase the heterogeneous performance (Nagai et al., 2004):

$$
\begin{aligned}
& f\left(f t_{\text {elem }}\right)=\frac{1}{\sqrt{2 \pi} \sigma} \exp \left[-\frac{\left(f t_{\text {elem }}-\mu\right)^{2}}{2 \sigma^{2}}\right] \\
& \mu=f t_{\text {average }} \\
& \sigma=-0.2 f t_{\text {average }}+1.5
\end{aligned}
$$

For the shear spring of porous body, the following criterion is adopted (Nagai et al., 2004):

$$
\tau_{\max }= \pm\left(0.11 t_{\text {elem }}^{3}\left(-\sigma+f_{\text {elem }}\right)^{0.6}+\mathrm{ft}_{\text {elem }}\right)\left(\sigma \leq \mathrm{ft}_{\text {elem }}\right)
$$

And for the interface between mortar and aggregate:

$$
\tau_{\max }= \pm(-\sigma \tan \phi+c)
$$

Since the internal stress is a volume stress, only the normal direction is considered for convenience. Therefore, finally the element stiffness matrix would be:

$$
k_{e}=\left[\begin{array}{cc}
k_{n}+k_{w} & 0 \\
0 & k_{s}
\end{array}\right]
$$

\section{NUMERICAL ANALYSIS}

\subsection{Simulation model}

The numerical model is conducted to simulate our previous experiments, that is, the saturated mortar (Sicat et al., 2013) and mortar with single aggregate (Sicat, Gong, Ueda, \& Zhang, 2014) in closed test, as well as the concrete column (Hasan et al., 2004) in open test. The 2D models are developed with the size and boundary conditions shown in Figure 12.

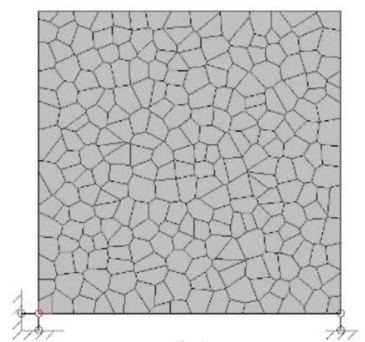

(a)

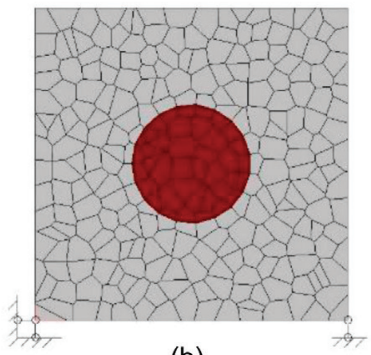

(b)

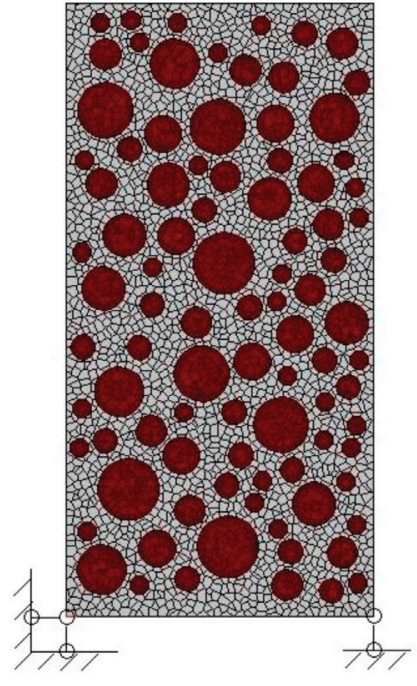

(c)
Figure 12. Simulation models and boundary conditions (a) mortar
$40 \mathrm{~mm} \times 40 \mathrm{~mm}$, (b) mortar with single aggregate $40 \mathrm{~mm} \times 40 \mathrm{~mm}$, (c) concrete $200 \mathrm{~mm} \times 100 \mathrm{~mm}$.

Table 2. Parameters in experiments and simulation.

\begin{tabular}{cccc}
\hline Parameters & Mortar & Aggregate & Interface \\
\hline$f_{t}(\mathrm{MPa})(\mathrm{a})$ & 5.67 & N.A. & 1.83 \\
$f_{t}(\mathrm{MPa})(\mathrm{b})$ & 4.09 & N.A. & 1.83 \\
$E(\mathrm{GPa})(\mathrm{a})$ & 34 & 50 & - \\
$E(\mathrm{GPa})(\mathrm{b})$ & 25 & 50 & - \\
$v$ & 0.18 & 0.25 & - \\
$c(\mathrm{MPa})$ & - & - & 3.05 \\
$\phi\left({ }^{\circ}\right)$ & - & - & 35 \\
$w_{\max }(\mathrm{mm})$ & 0.03 & N.A. & 0.01 \\
\hline
\end{tabular}

Note: (a) for closed test (Sicat et al., 2013, 2014); (b) for open test (Hasan et al., 2004). N.A. means the aggregate is assumed not fracture. $w_{\max }$ is the maximum crack width.

The input material properties are chosen according to those experiments and also some are from the empirical values (Table 2). Figure 12(a) and (b) are for the closed test under 30 freeze-thaw cycles, with the cooling rate of $15^{\circ} \mathrm{C} / \mathrm{h}$ and lowest temperature of $-28^{\circ} \mathrm{C}$. Figure 12 (c) is for the open test under 300 cycles, and the cooling rate is around $7.5^{\circ} \mathrm{C} / \mathrm{h}$ with lowest temperature of $-20^{\circ} \mathrm{C}$.

Here, the temperature and moisture distribution are assumed uniform because the size of specimens are small ( $40 \mathrm{~mm} \times 40 \mathrm{~mm} \times 2 \mathrm{~mm}$ in Sicat's test). And even for bigger specimens, in Hasan's test, the measured temperatures at different locations inside the concrete column still showed uniform distribution. Therefore, the moisture movement and temperature difference are ignored in the simulation model, and once the environmental conditions are given, the internal stress should be the same on all the normal springs, but only exists in the mortar and interfaces, because the ice formation in the aggregates is neglected.

The internal stresses are estimated according to the physical model discussed in Section 2. Once knowing the internal pore pressure $\left(p_{\mathrm{h}}+p_{\mathrm{l}}+p_{\mathrm{c}}\right)$, the equivalent internal stress can be calculated as:

$$
\sigma=\frac{2 \phi}{1+\phi}\left(p_{h}+p_{l}+p_{c}\right)
$$

For the closed test of Sicat et al. (2013, 2014), the moisture condition is known and it is easier to calculate the time-dependent stress. But here, since the damage is mainly considered as the peak strain under peak stress and also the unrecoverable deformation (residual strain), then only the peak values of internal stresses are given to the program. Figure 13(a) shows the estimated input stresses in the closed test. The hydraulic pressure is the highest at the beginning but gradually reduced as damage cumulates. Finally there would only be the negative stress due to cryosuction. However, for the open test 
of Hasan et al. (2004), the specimens are bigger and not saturated at the beginning. Due to the continuously moisture supply, the saturation degree would increase and result in increasing hydraulic pressure. The water uptake measured by Fagerlund (2002) is increasing with the number of cycles but with decreasing speed. Therefore, same increasing character of input stress is assumed for open test [Fig. 13 (b)]. Since the exact values of internal stress are difficult to measure, after knowing the tendency of Figure 13 qualitatively, the proper magnitudes are chosen to ensure a better match with experimental deformations.
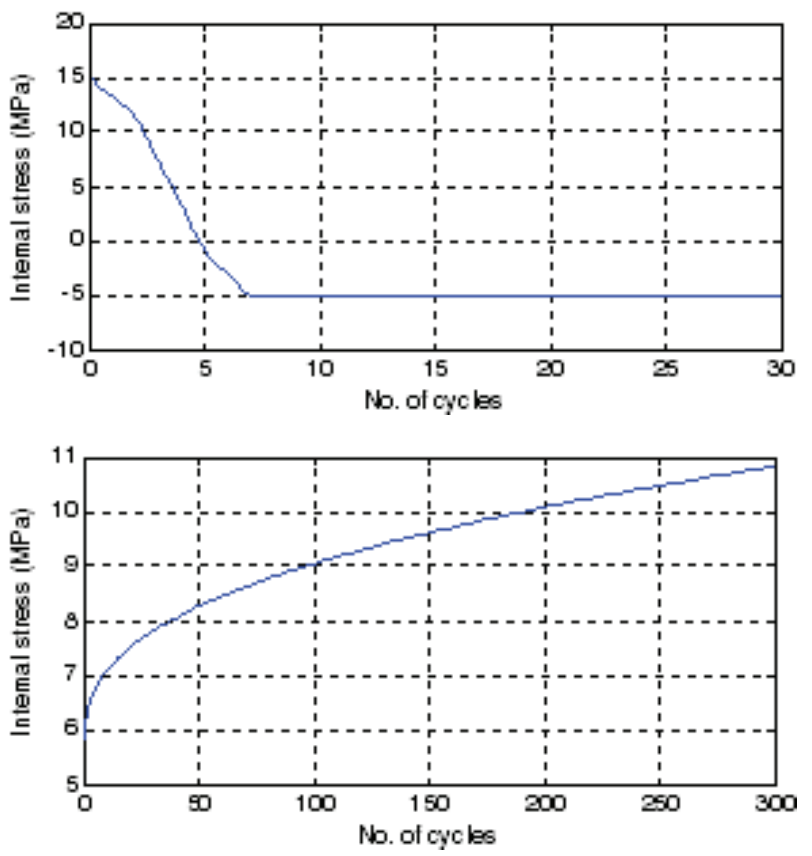

Figure 13. Estimated input of total internal stresses (a) for closed test (b) for open test.

\subsection{Results and discussions}

Using the input stress from Figure13(a), the mortar deformation in 30 cycles are simulated; see Figure 14. Since the tension-softening behavior is considered here, the result fits better to the experiment than the linear poroelastic model [Figure 6(b)]. Figure 15 shows the deformation of mortar, aggregate, and interface separately. The interface deformation is compared with Sicat et al.'s data (2014), the cumulated shrinkage in the experiment is believed as creep, but here it is not included in the current model. However, if comparing the net deformation of each cycle, the calculated and experimental results are still similar.

The deformation of mortar part in Figure $15(b)$ is smaller than that of pure mortar (Figure 14). This is because the existence of aggregate can be regarded as an additional displacement boundary, then the mortar will deform less due to this new restriction. However, at the same time, the interface is usually thought as the weak point, so the incompatibility of deformation between mortar and aggregate will cause a big burden on the interface and results in much bigger cracking [Figure 15(a)]. Finally, the aggregate deforms elastically due to the stress on the interface [Figure 15(c)].

The simulation results of concrete column are shown in Figure 16. It can be seen that the crack opening of interface is much larger than the mortar part; also see Figure 17, which shows the crack opening at the 100th cycle. From Figures 16 and 17, it can also be seen that bigger aggregates have larger crack width, and since the left half of the model contains a bigger aggregate, it will deform more, and finally the specimen will shift slightly to the right.

Figure 18 shows the comparison between the simulated and experimental axial strain. Still only the peak and residual strain are calculated of each cycle, and it can be seen that the residual strain matches the experiments well. In Hasan et al.'s paper (2004), other material property degradations such as elastic modulus, and compressive strength are related to the residual strain (or plastic tensile strain); therefore, once the simulation model can fit the residual strain well, it can also predict other property changes, which will be investigated in the future.

\section{CONCLUSIONS}

In this paper, the deformational behavior of mortar and concrete suffering freeze-thaw cycles are theoretically investigated and numerically simulated. Both the physical model and the numerical analysis show satisfactory agreements with previous closed and open freeze-thaw tests. And some useful remarks are concluded:

(1) According to the moisture condition, the internal stress could be either positive or negative, and also, during large number of freeze-thaw cycles, the deformation could be either increasing to expansion or reversing to contraction, and therefore, the hydraulic pressure model presented in this paper, which combines Powers' model and poromechanical theories has more flexibility to be applied to different cases.

(2) The hydraulic pressure itself varies with the material's deformation, but it can be transformed into a constant value applied on the parallel spring system, which can become much easier for numerical analysis, such as FEM and RBSM. And also by doing so, the internal pressure can be applied together with external loadings, then the combined internal and external effect could be simulated, which will be conducted in the next step.

(3) The simulation results show that the mortaraggregate interface is the most vulnerable part, 


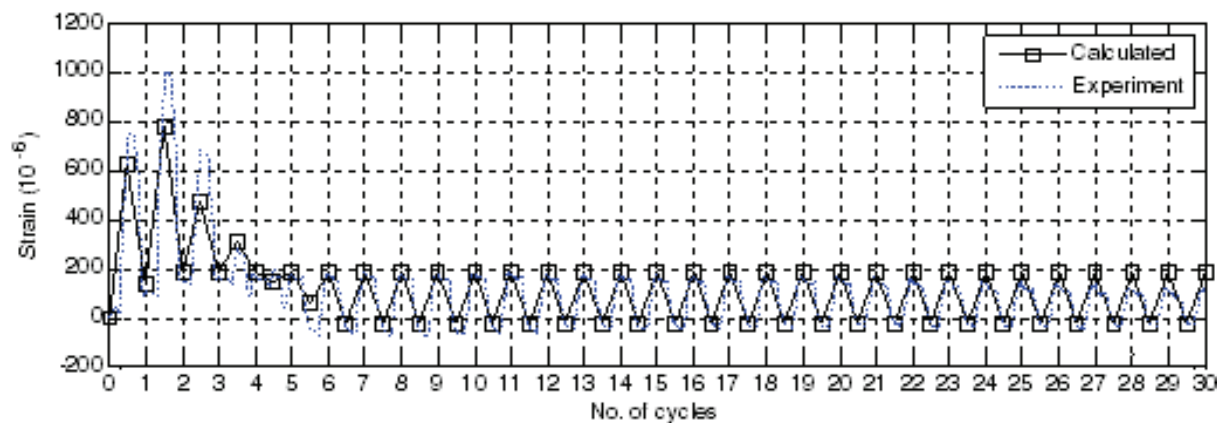

Figure 14. Comparison between simulated and experimental deformation [pure mortar, Figeur12 (a)].

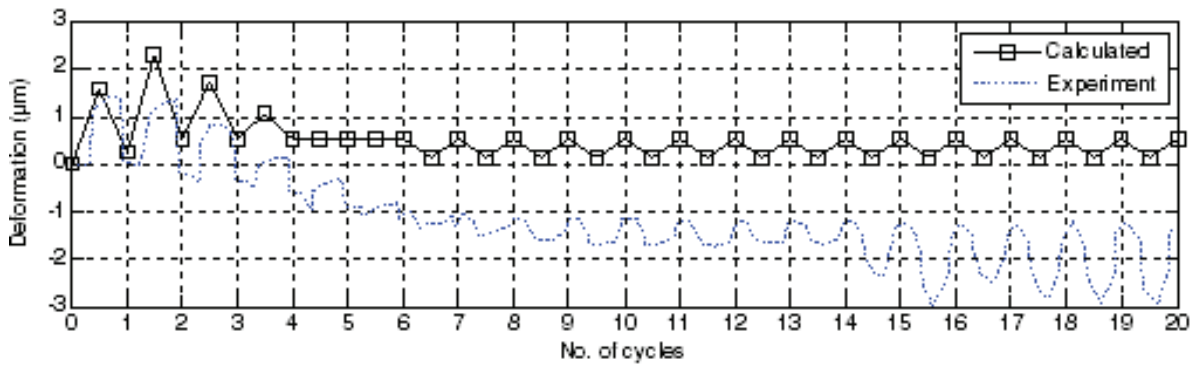

(a)

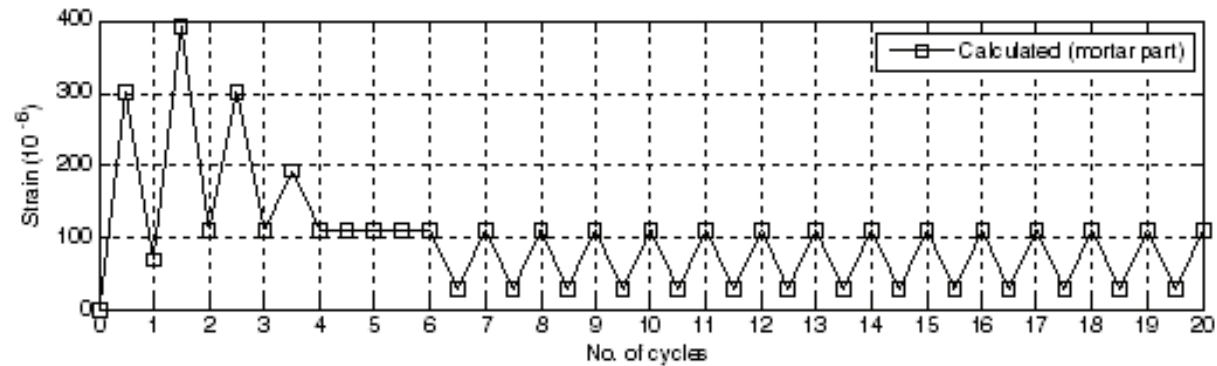

(b)

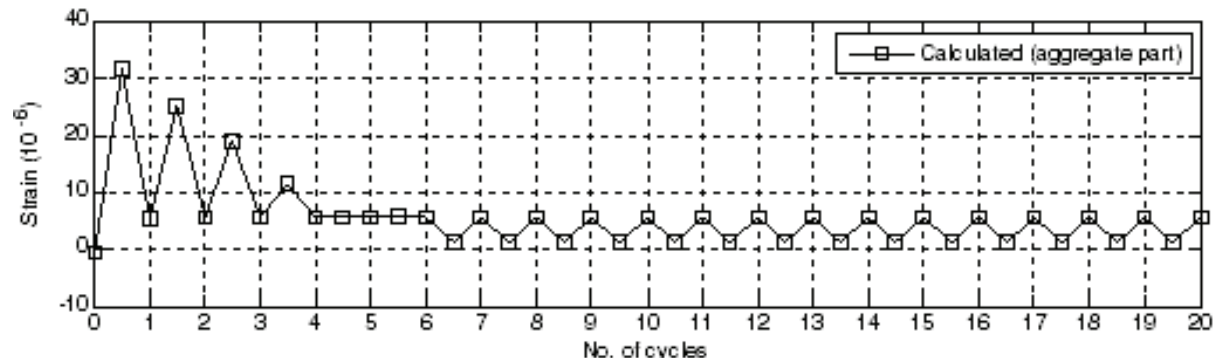

(c)

Figure 15. Comparison between simulated and experimental deformation [mortar with single aggregate, Figure 12 (b)]; (a) mortar-aggregate interface, (b) surrounding mortar, (c) aggregate.
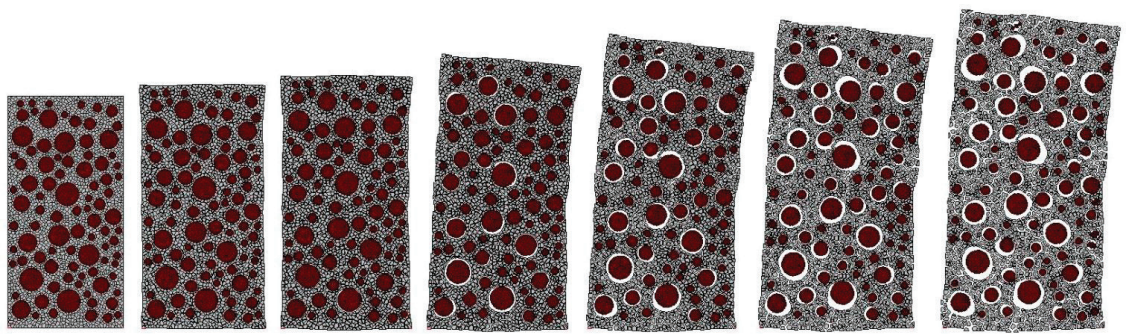

Figure 16. Simulated and experiment data of concrete under 300 cycles in open test (every 50 cycles from 0 to 300 , deformation is enlarged by 200 times). 
and its deformation is mainly due to the deformation incompatibility between mortar and aggregate; bigger aggregate results in larger crack opening.

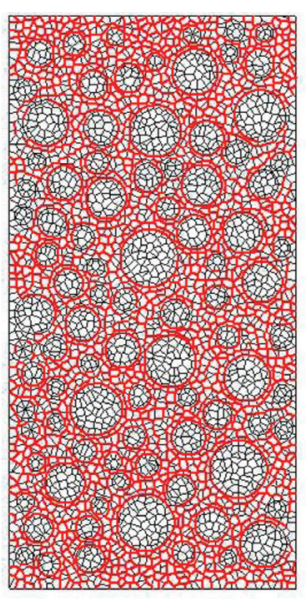

(a)

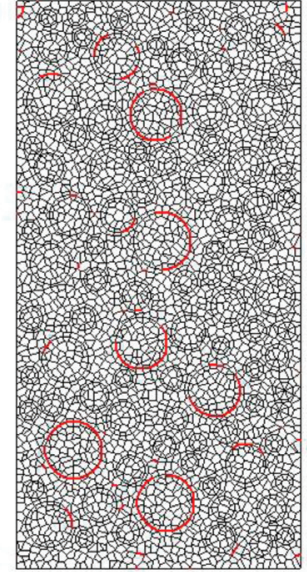

(b)
Figure 17. Crack width at 100th cycle (a) reaches $0.002 \mathrm{~mm}$, (b) reaches $0.005 \mathrm{~mm}$.

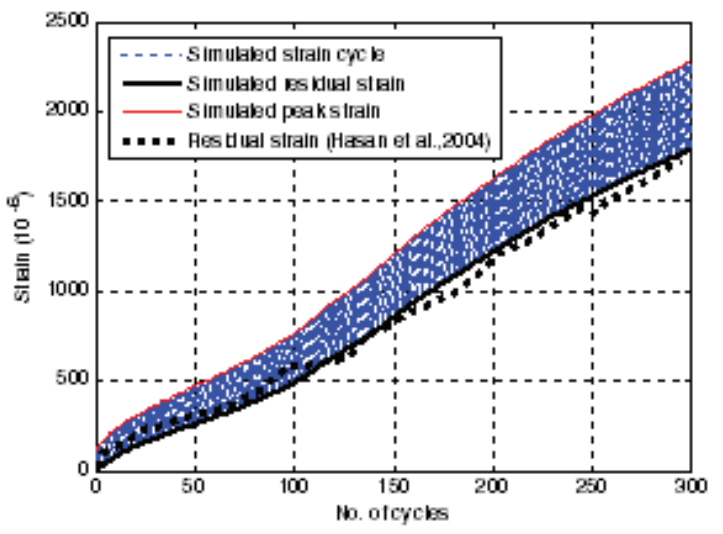

Figure 18. Simulated and experiment data of concrete under 300 cycles in open test.

\section{REFERENCES}

Coussy, O. (2004). Poromechanics. West Sussex, England: Wiley.

Coussy, O. (2005). Poromechanics of freezing materials. Journal of the Mechanics and Physics of Solids, 53, 1689-1718.

Coussy, O., \& Monteiro, P. J. M. (2008). Poroelastic model for concrete exposed to freezing temperatures. Cement and Concrete Research, 38, 40-48.

Fagerland, G. (2002). Mechanical damage and fatigue effects associated with freeze-thaw of materials. In M. J. Setzer, R. Auberg, and H.-J. Keck (eds.), Proceedings of the 2nd international RILEM workshop on frost resistence of concrete (pp. 117-132). Bagneux, FR: RILEM.

Gong, F., Sicat, E., Ueda, T., \& Zhang, D. (2013a). Meso-scale mechanical model for mortar deformation under freeze-thaw cycles. Journal of Advanced Concrete Technology, 11, 49-60.

Gong, F., Zhang, D., Sicat, E., \& Ueda, T. (2014). Empirical estimation of pore size distribution in cement, mortar, and concrete. Journal of Materials in Civil Engineering, 26(7), 04014023. http://dx.doi. org/10.1061/(ASCE)MT.1943.5533.0000945.

Hasan, M., Okuyama, H., Sato, Y., \& Ueda, T. (2004). Stress-strain model of concrete damaged by freezing and thawing cycles. Journal of Advanced Concrete Technology, 2(1), 89-99.

Kaufmann, J. P. (2002). A qualitative sequential frost deicing salt damage model based on experimental data. Proceedings of the 2nd international RILEM workshop on frost resistence of concrete (pp. 197-204). Bagneux, FR: RILEM.

Kawai, T. (1977). New element models in discrete structure analysis. Journal of Society of Naval Architectures of Japan, 141, 187-193.

Matsumoto, K., Sato, Y., Ueda, T., \& Wang, L. (2008). Mesoscopic analysis of mortar under high-stress creep and low-cycle fatigue loading. Journal of Advanced Concrete Technology, 6(2), 337-352.

Nagai, K., Sato, Y., \& Ueda, T. (2004). Mesoscopic simulation of failure of mortar and concrete by $2 \mathrm{D}$ RBSM. Journal of Advanced Concrete Technology, 2(3), 359-374.

Powers, T. C. (1949). The air requirement of frostresistant concrete. Proceedings of Highway Research Board, 29, 184-211.

Scherer, G. W., \& Valenza, J. J. II (2005). Mechanism of frost damage. In J. Skalny \& F. Young (Eds.), Materials science of concrete (Vol. VII), 209-246, Westerville, $\mathrm{OH}$ : American Ceramic Society.

Sicat, E., Gong, F., Ueda, T., Zhang, D. (2014). Experimental investigation of the deformational behavior of the interfacial transition zone (ITZ) in concrete during freezing and thawing cycles. Construction and Building Materials, 65, 122-131. http://dx.doi.org/10.1016/j. conbuildmat.2014.04.035.

Sicat, E., Gong, F., Zhang, D., \& Ueda, T. (2013). Change of the coefficient of thermal expansion of mortar due to damage by freeze-thaw cycles. Journal of Advanced Concrete Technology, 11, 333-346.

Sun, Z., \& Scherer, G. W. (2010a). Effect of air voids on salt scaling and internal freezing. Cement and Concrete Research, 40, 260-270.

Ueda, T., Hasan, M., Nagai, K., Sato, Y., \& Wang, L. (2009). Mesoscale simulation of influence of frost damage on mechanical properties of concrete. Journal of Materials in Civil Engineering, 21(6), 244-252.

Yang, Z., Weiss, W. J., \& Olek, J. (2006). Water transport in concrete damaged by tensile loading and freeze-thaw cycling. Journal of Materials in Civil Engineering, 18(3), 424-434. 